\title{
Space Target Identification based on RBF Neural Network Ensembles
}

\author{
Jubo Zhao \\ 83 Unit 92941 Troops, PLA \\ Huludao City, Liaoning Province \\ China, 125001 \\ zhaojubo5151@gmail.com
}

\begin{abstract}
Space target identification is very important to space security. In practice, identification of satellites from space images is very useful and difficult. After preprocessing of space images, the object in the space images need to be classified as satellites or not using classification algorithms. In this paper, we propose to use RBF neural network ensemble to identify satellites in the space images. The simulation system for space target identification is constructed; the total identification process is tested and the experiment results are analyzed. The correct identification ratio is larger than $90 \%$ when there are three satellites in the system, and the correct ratio is about $85 \%$ when there are six satellites. These results demonstrate that this method has a promising identification effect.
\end{abstract}

Keywords- neural network ensembles; RBF neural networks; target identification; space target identification

\section{INTRODUCTION}

The detection and identification techniques are playing an important role in the application fields such as space resources exploitation and space security. By analyzing the space target images that are captured by space-based optical-electronic surveillance systems, the shape, size, functions and type of space target could be judged and obtained [1] by analyzing the images. However, many factors would influence the accuracy of identification based on these space-based images. For example, a) the gesture of target is changing and these objects in the space often change the gesture; b) the observation distance is far, and 3) the background in these space images is complicated with a lot of background noise. All these factors will change the target gesture views, scales and image's brightness and contrast, etc. Therefore, the space target identification problems are too complex to be solved effectively $[2,3,4]$.

In this paper, we propose a hierarchical identification algorithm by introducing the two-stage matching procedure, i.e. raw matching and refined procedures. The hierarchical algorithm will generate a candidate model library by raw matching in the first stage, and then use this library to train ensemble models by extracting the features from these models. The algorithm operates in an online manner, so the algorithm runs fast.

At present, the popular artificial neural network (NN) models that are widely used include backward propagation (BP) neural network, radial basis function (RBF) neural network and so on $[5,6,7]$. The training of BP neural network is not very effective, thus it is inappropriate for this application. RBF neural networks are more effective in training. However, there are many uncertain factors, including parameter settings, the number of hidden nodes, in the training of RBF neural networks. .

Ensemble learning approaches benefit from combining multiply classifiers and these approaches are robust to parameter settings. Therefore, in this paper we propose to employ ensemble of RBF neural networks for the space target identification. The contributions of this paper include a) the algorithm is an effective and efficient online processing algorithm in the space target identification; b) the two-stage algorithm operates in a hierarchical manner, which saves the computational complexity. c) the simulation system has been implemented and tested with accurate predictions.

\section{IDENTIFICATION METHOD BASED ON THE RBF NEURAL NETWORK ENSEMBLES}

\section{A. Details of RBF Networks}

The component network in the ensemble is a radial basis function (RBF) network. The output of RBF network is computed as a linear combination of $n_{i}$ basis functions

$$
f_{i}(x)=\sum_{k=1}^{n_{i}} w_{k} \phi_{k}(x)=\Phi^{T} w_{i}
$$

where $w_{i}=\left(w_{1}, \ldots, w_{n_{i}}\right)$ denotes the weight vector in the output layer and $\Phi=\left(\phi_{1}, \ldots, \phi_{n_{i}}\right)$ is the vector of basis functions. The Gaussian basis functions $\phi_{k}$ are defined as

$$
\phi_{k}=\exp \left(\frac{\left\|x-\rho_{k}\right\|^{2}}{2 \sigma_{k}^{2}}\right)
$$

where $\rho_{k}$ and $\sigma_{k}$ denote the mean and width of the Gaussian, respectively. The training of RBF network is separated into two steps. In the first step, the means $\rho_{k}$ are initialized with randomly selected data points from the training set and the variances $\sigma_{k}$ are determined as the Euclidean distance between $\rho_{k}$ and the closest $\rho_{i}\left(i \neq k, i \in\left\{1, \ldots, n_{i}\right\}\right)$. Then in the second step we perform a gradient descent in the regularized error function (weight decay)

$$
\min e_{i}=\frac{1}{M} \sum_{n=1}^{N}\left(f_{i}\left(x_{n}\right)-y_{n}\right)^{2}+\alpha_{i} \sum_{k=1}^{n_{i}} w_{k}^{2}
$$


The derivative of the above Equation with respect to $w_{k}$ is:

$$
\frac{\partial e_{i}}{\partial w_{k}}=\frac{2}{M} \sum_{n=1}^{N}\left(f_{i}\left(x_{n}\right)-y_{n}\right) \frac{\partial f_{i}\left(x_{n}\right)}{\partial w_{k}}+2 \alpha_{i} w_{k}
$$

In order to fine-tune the centers and widths, we simultaneously adjust the output weights and the RBF centers and variances. Taking the derivative of error function with respect to RBF means $\rho_{k}$ and variances $\sigma_{k}^{2}$ we obtain

$$
\frac{\partial e_{i}}{\partial \rho_{k}}=\frac{2}{M} \sum_{n=1}^{N}\left(f_{i}\left(x_{n}\right)-y_{n}\right) \frac{\partial f_{i}\left(x_{n}\right)}{\partial \rho_{k}}
$$

with $\frac{\partial f_{i}\left(x_{n}\right)}{\partial \rho_{k}}=w_{k} \frac{x_{n}-\rho_{k}}{\sigma_{k}^{2}} \phi_{k}\left(x_{n}\right)$ and

$$
\frac{\partial e_{i}}{\partial \sigma_{k}}=\frac{2}{M} \sum_{n=1}^{N}\left(f_{i}\left(x_{n}\right)-y_{n}\right) \frac{\partial f_{i}\left(x_{n}\right)}{\partial \sigma_{k}},
$$

with $\frac{\partial f_{i}\left(x_{n}\right)}{\partial \sigma_{k}}=w_{k} \frac{\left\|x_{n}-\rho_{k}\right\|^{2}}{\sigma_{k}^{3}} \phi_{k}\left(x_{n}\right)$. These three derivatives are employed in the minimization of error function by a scaled conjugate gradient descent algorithm.

\section{B. RBF Neural Network Ensemble}

Ensembles of multiple learning machines, i.e. groups of learners that work together as committees; have attracted a lot of research interest in the machine learning community since this method is considered as a good approach to improve the generalization ability. The term "ensemble" can be used to describe the paradigm that brings together a number of learning machines for the same task. This technique originates from Hansen and Salamons' work [9], which showed that the generalization ability of a neural network can be significantly improved through ensembling a number of neural networks. Because of the simple and effective properties, ensemble research has become a hot topic in the machine learning community and has already been successfully applied to many areas, for example face recognition [10], character recognition [11], image analysis [12], etc.

In RBF neural network ensembles there are many uncertain factors. For example, the parameter settings play an important role in the ensemble. To reduce the influence of parameters, we introduce RBF neural network ensembles. The sketch map of neural network ensemble is shown in Figure 1.

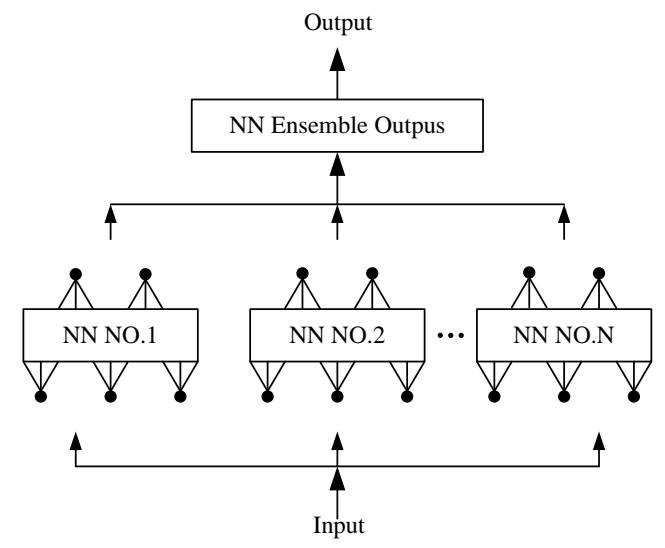

Figure 1. Sketch map of neural networks ensemble

It can be seen that the constructing methods of individual neural network and the integration methods of the outputs are the core contents of the neural network ensemble research. In this paper, by setting different RBF parameters, including the number of hidden nodes, different individual RBF neural networks can be generated. The major voting strategy is employed in the ensemble integration.

After incorporating ensemble of learning machines with major voting, the candidate model library can be used to train the ensemble. Then, the trained ensemble model will be employed for identification of space target.

The flow chart of identification progress based on the RBF neural network ensemble is shown in Figure 2. In practical applications, there could be some problems. we try to generate an ensemble with odd number of ensemble members to avoid the situations when two output classes have the same vote.

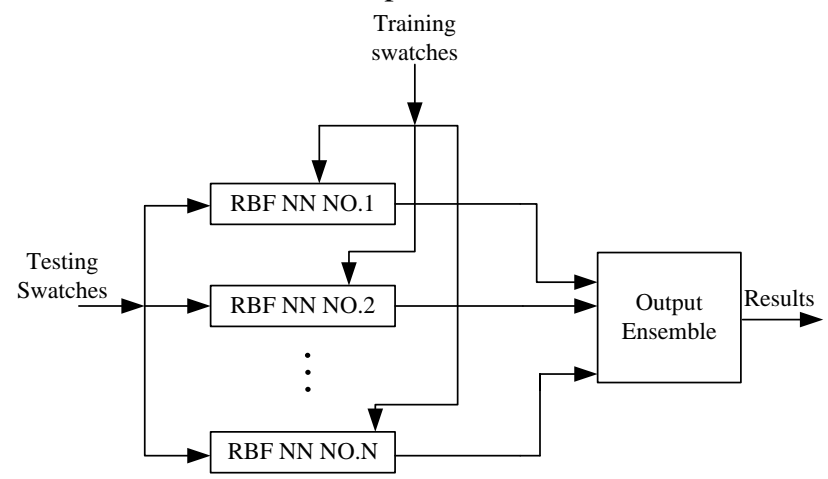

Figure 2. Flow chart of identification progress

\section{Flowchart of Space Target Identification}

The space target identification system can be divided into four subsystems, including image acquirement subsystem, image preprocessing subsystem, feature extraction subsystem and classifying identification subsystem, as shown in Figure 3. We use modeling tool software 3DSMax to build 3D models of satellites and obtain their multi-viewing point side elevation images to realize image acquirement subsystem [8]. 
The raw image data consists of a lot of background noise, which must be removed. The corresponding procedures, including background removal and wavelet denoise are required to pre-process the image data.

Background removal will remove some consistent noise. A median filter is performed on the GPR data to remove the noise. Wavelet denoise is to remove some non-constant noise in the image data. Meanwhile, the image enhancement techniques are employed in this application to clearly identify these objects in the images.

The edge detection algorithms are employed in this paper to identify the edge for each object in these images.

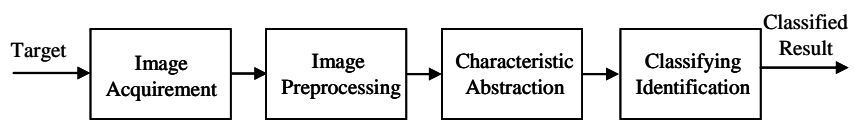

Figure 3. Target identification system

The general procedure of target identification is as follows. First, we preprocess the "satellite"-like images and generate binary images, then judge whether targets in the images are axisymmetric or not. If the target is axisymmetric, choose the first rough matching method, otherwise choose the second rough matching method. The first rough matching method is able to process axisymmetric satellite images by comparing the test satellite image with the images in the library with different views and different angles based on the principal-axis angles.

The second rough matching method is able to process satellite images that are not axisymmetric. It compares the test satellite image with all images in the library with different views/angels. The algorithm also measures the scale/size of the satellite to estimate the distance to facilitate the target identification. After the rough matching stage, we will compare the target images with the candidate models in the library by using the RBF neural network ensemble, and get the identification results

\section{EXPERIMENTAL STUDY}

Based on the actual shape parameters of satellites, we use modeling tool 3DSMax to build eight satellite models, as shown in Figure 4. The indexes of satellites are T1、T2、 T3、T4、T5、T6、T7 and T8, respectively.
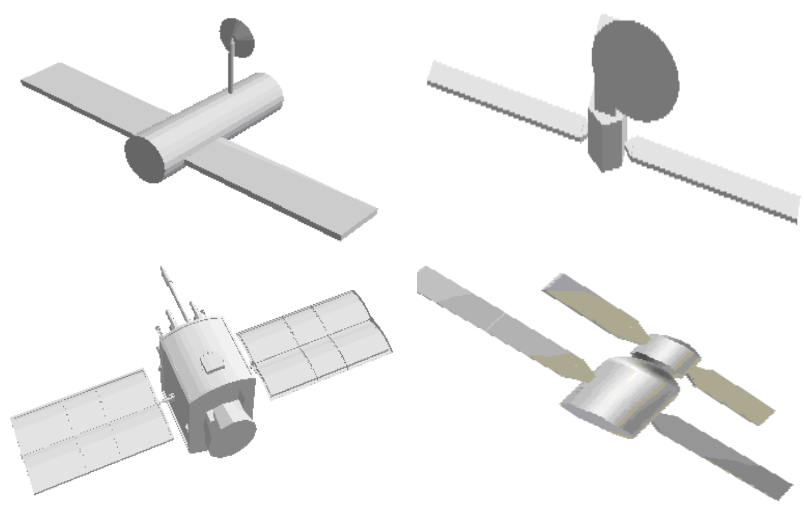
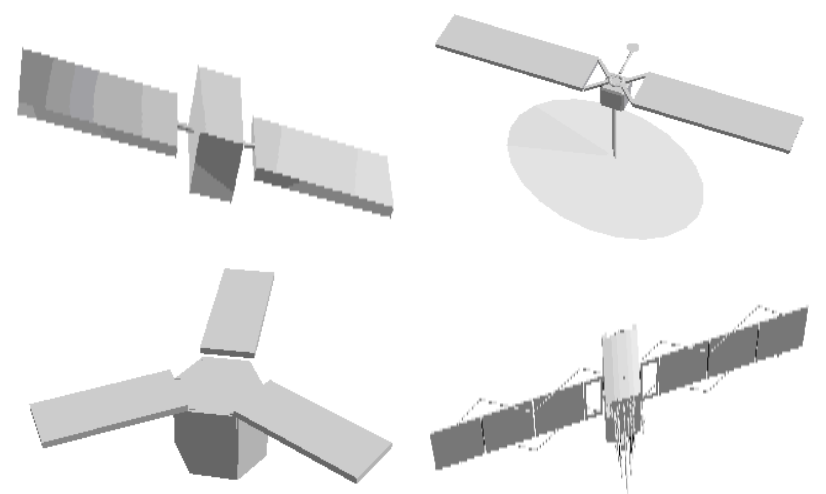

Figure 4. 3D satellites models

The experiment is conducted by using MATLAB 7.0, and the training procedures are shown as follows:

(1) The training target (error precision) of single RBF networks is 0.1 , the spread of radial basis function is chosen within the range $[1,3]$ by the interval of 0.5 .

(2) If the output classes can't be determined after one training, perform the training again and change the spread of radial basis function to $(0.8,1,1.5,1.6,2,2.4,2.5,3)$.

\section{A. Identification Results of Three Satellites}

In this section, we report the experimental results using three satellites. We choose three satellite models randomly, in the scope that the pitching angle is changing form $-90^{\circ}$ to $90^{\circ}$, and the azimuth angle is changing form $0^{\circ}$ to $360^{\circ}$. The images are captured in the interval of $20^{\circ}$ based on the method of observing sphere which is carved up by longitude and latitude. Thus, we obtain 162 different viewing images for each satellite, and there are 486 images for three satellites in total. The size is $256 \times 256$ for each image. These 486 images are used as training set by processing these images after rough match stage, and then fed the processed images/data into neural network ensemble.

In the obtained satellite models, the test set with 864 images is generated by sampling in the interval of $15^{\circ}$ and each image's size is also $256 \times 256$.

These 864 images will be processed in the first rough matching stage and then we employ the training neural network ensembles to calculate the identification ratio. In this section, we report a demonstration with three satellites whose indexes are T7, T3, T6, respectively. The scale of the satellite in these images is chosen as 1:1. By using the proposed methods described above, we obtain the training set and testing set. The identification results are shown in Table 1. For example, there are 270 images of satellite T3 that are classified correctly, and 18 images that are mis-classified as satellite T7. The correct identification ratio of $\mathrm{T} 3$ is $93.75 \%$. 
TABLE I. IDENTIFICATION RESULTS OF 3 SATELLITES (SCALE IS 1)

\begin{tabular}{|c|c|c|c|c|c|c|}
\hline Satellite & T7 & T3 & T2 & Uncertain & $\begin{array}{c}\text { Incorrect } \\
\text { output }\end{array}$ & $\begin{array}{c}\text { Correct } \\
\text { ratio }\end{array}$ \\
\hline $\mathbf{T 7}$ & 288 & 0 & 0 & 0 & 0 & $100 \%$ \\
\hline T3 & 18 & 270 & 0 & 0 & 0 & $93.75 \%$ \\
\hline T2 & 0 & 8 & 272 & 8 & 0 & $94.45 \%$ \\
\hline
\end{tabular}

Assume that the distance of the satellite is changed, the scale of the satellite image will be changed to realize the effect. We keep the training set, and change the satellite scale of test set from 1 to 0.8 , and the identification results is shown in Table 2.

Again, we keep the training set and change the satellite scale of test set from 1 to 1.2. The results are shown in Table 3 .

According to Tables 1, 2 and 3, the identification results of this method is promising; especially this algorithm can process the satellite images with different views/angels. The distance, i.e. the scale, of the satellite in these images does not have much impact on the identification result. In addition, we have also tried other 10 combinations of three satellites, and almost all the identification ratios are larger than $90 \%$.

TABLE II. IDENTIFICATION RESULTS OF 3 SATELLITES (SCALE IS 0.8)

\begin{tabular}{|c|c|c|c|c|c|c|}
\hline Satellite & T7 & T3 & T2 & Uncertain & $\begin{array}{c}\text { Incorrect } \\
\text { output }\end{array}$ & $\begin{array}{c}\text { Correct } \\
\text { ratio }\end{array}$ \\
\hline T7 & 277 & 9 & 2 & 0 & 0 & $96.18 \%$ \\
\hline T3 & 14 & 269 & 0 & 0 & 5 & $93.4 \%$ \\
\hline T2 & 1 & 14 & 265 & 8 & 0 & $92.01 \%$ \\
\hline
\end{tabular}

TABLE III. IDENTIFICATION RESUlTS OF 3 SATELLITES (SCALE IS 1.2)

\begin{tabular}{|c|c|c|c|c|c|c|}
\hline Satellite & T7 & T3 & T2 & Uncertain & $\begin{array}{c}\text { Incorrect } \\
\text { output }\end{array}$ & $\begin{array}{c}\text { Correct } \\
\text { ratio }\end{array}$ \\
\hline T7 & 280 & 8 & 0 & 0 & 0 & $97.22 \%$ \\
\hline T3 & 15 & 270 & 0 & 0 & 3 & $93.75 \%$ \\
\hline T2 & 0 & 10 & 267 & 10 & 1 & $92.71 \%$ \\
\hline
\end{tabular}

TABLE IV. IDENTIFICATION RESULTS OF 6 SATELLITES

\begin{tabular}{|c|c|c|c|c|c|c|c|c|c|}
\hline Satellite & T7 & T3 & T2 & T8 & T1 & T6 & Uncertain & $\begin{array}{c}\text { Incorrect } \\
\text { output }\end{array}$ & $\begin{array}{c}\text { Correct } \\
\text { ratio }\end{array}$ \\
\hline T7 & 246 & 32 & 0 & 0 & 0 & 0 & 10 & 0 & $85.42 \%$ \\
\hline T3 & 15 & 239 & 11 & 0 & 8 & 0 & 7 & 8 & $82.99 \%$ \\
\hline T2 & 0 & 8 & 262 & 12 & 0 & 0 & 0 & 6 & $90.97 \%$ \\
\hline T8 & 8 & 38 & 14 & 228 & 0 & 0 & 0 & 0 & $79.17 \%$ \\
\hline T1 & 0 & 0 & 0 & 14 & 262 & 6 & 2 & 4 & $90.97 \%$ \\
\hline T6 & 0 & 0 & 0 & 0 & 24 & 254 & 10 & 0 & $88.19 \%$ \\
\hline
\end{tabular}

\section{B. Identification Results of Six Satellites}

The experimental settings for six satellites are presented as follows. For each satellite, we capture images in the interval of $20^{\circ}$ and obtain 162 different viewing angle images. In total, there will be 972 images for six satellites. The size of each image is $256 \times 256$. The generated 972 images will be used as training set. By using the rough matching procedure and feature selection, these processed data is taken to the RBF neural network ensemble. The test set is generated by capturing the satellites images with different views/angles in the interval of $15^{\circ}$, and finally we obtain 1728 images in total. These 1728 images will be treated as testing set.
Here, we choose six satellites, T7、T3、T2、T8、T1 and T6, to do a demonstration. By using the methods described above, we obtain the training and testing sets, and the identification results are shown as Table IV.

According to Table 4, it is easy to see that although the identification ratios of six satellites with different viewing points are lower than the identification ratios of three satellites, the ratios are still satisfactory (about 85\%). The identification ratios are decreasing because: 1) First, there are more satellites in the library and the size of training set is not increased significantly; 2)Second, many satellites are similar as each other in terms of the shape as the satellite numbers are increasing, and in the same viewing point there are more images of different satellites look similar. The two reasons cause the relatively lower identification results. 


\section{CONCLUSIONS}

In general, the identification approach based on the RBF neural network ensemble has a nice identification effect and the approach can recognize satellite targets correctly with a high identification ratio.

The identification of satellite targets is complicated with many open questions. There are still many problems to solve in the future. To improve the identification ratio of satellite identification, some further work will be conducted. For example, by combining satellites' orbiting parameters, observable scopes and other information synthetically, we obtain much more satellite images of multi-viewing points in the model library, and the approach could increase the numbers of satellites to identify and keep less training points at the same time. On the other hand, we could combine the information of orbit parameters and imaging distance to identify targets with the same training set.

\section{REFERENCES}

[1] Jayant S, "Space-based visible space surveillance performance," Journal of Guidance, Control and Dynamics, vol. 23, pp.153-158, 2000

[2] Qin Lei, Liu Wen bin , Zhou Kang, “An Information Fusion Based on Group of Neural Network to Recognize Spatial Targets," Microel Ectronics \& Computer, vol 25, pp. 117-120. 2008

[3] Tao Xiaoli, Howard E.Michel, "Classification of multispectral satellite image data using improved NRBF neural networks," Intelligent Robots and Computer Vision XXI: Algorithms, Techniques, and Active Vision, Proceedings of SPIE SPIE, vol. 5267, pp. 311-320, 2003
[4] Zhang Jian, Zhou Xiaodong, Zhang Shifeng, Gou Lei, "Recognition of Satellite Targets Based on Combined Invariant Moments and Artificial Neural Network", Journal of Naval Aeronautical Engineering Institut, vol. 23, pp. 29-32, 2008

[5] Dan Xu, Peng Feiyu, "Palmprint recognition based on modified DCT features and RBF neural network," IEEE Machine Learning and Cybernetics. Kunming, China, vol pp. 2982-2986, 2008

[6] Yu Xinghuo, Onder M Ere, Okyay Kaynak, "A general backpropagation algorithm for feedforward neural networks learning”. IEEE Transactions on neural networks. Qld., Australia, vol. 13(1), pp. 251254, 2002

[7] Antreas Afantitis, Georgia Melagraki, Kalliopi Makridima, Alex Alexandridis, Haralambos Sarimveis, Olga Iglessi Markopoulou, "Prediction of high weight polymers glass transition temperature Using RBF neural networks," Journal of Molecular Structure, vol. 716, pp. 193-198, 2005

[8] Luo Yuan, Ma Huimin, Li Fengting. "3D object recognition technique based on muti-resolution aspect graph," IEEE Neural Networks and Brain. Beijing, China, pp. 1168-1172, 2005.

[9] L. K. Hansen and P. Salamon, "Neural network ensembles," IEEE Transactions on Pattern Analysis and Machine Intelligence, vol. 12, no. 10, pp. 993-1001, 1990.

[10] F. J. Huang, T. Chen, Z. Zhou, and H. Zhang, "Pose invariant face recognition," in Proceedings of the Fourth IEEE International Conference on Automatic Face and Gesture Recognition 2000, Washington, DC, USA, 2000, pp. 245-250.

[11] L. K. Hansen, L. Liisberg, and P. Salamon, "Ensemble methods for handwritten digit recognition," in Proceedings of the IEEE Workshop on Neural Networks for Signal Processing, Helsingoer, Denmark, 1992, pp. 333-342.

[12] K. J. Cherkauer, "Human expert level performance on a scientific image analysis task by a system using combined artificial neural networks," in Proceedings of AAAI-96 Workshop on Integrating Multiple Learned Models for Improving and Scaling Machine Learning Algorithms, Menlo Park, CA, USA, 1996, pp. 15-21. 